\title{
A Current Approach to Hyperprolactinemia
}

\author{
Lau $C S^{1}$ and $A w T C^{1,2^{*}}$ \\ ${ }^{1}$ Department of Laboratory Medicine, Changi General Hospital, Singapore \\ ${ }^{2}$ Department of Medicine, National University of Singapore, Singapore
}

*Corresponding author: Tar Choon Aw, Department of Laboratory Medicine, Changi General Hospital, Simei Street 3, 529889, Singapore, Tel: +65-68504927, Fax: +65-64269507, E-mail: tarchoon@gmail.com

\begin{abstract}
Prolactin is a hormone secreted by the anterior pituitary lactotrophs and is controlled by the influence of hypothalamic dopamine. PRL stimulates the proliferation and differentiation of mammary cells for lactation. There are many causes for hyperprolactinemia, including analytical, physiological, pharmacological, pathological and idiopathic causes. Macroprolactin is a significant analytical cause of hyperprolactinemia, and the most common cause of pathological hyperprolactinemia is prolactin secreting adenomas. Symptoms of true hyperprolactinemia include galactorrhoea, secondary amenorrhea, oligomenorrhea and hypogonadotropic hypogonadism. Larger macroadenomas can cause compressive symptoms on the pituitary gland or visual symptoms. A single measurement of serum PRL obtained at any time of the day can be used to diagnose hyperprolactinemia. Macroprolactinemia should be excluded in all cases of hyperprolactinemia, and polyethylene glycol precipitation is a fast, convenient and inexpensive method to exclude macroprolactinemia. Other interferents that can cause falsely low prolactin readings include the hook effect, biotin and heterophilic antibodies. Physicians must know how to approach hyperPRL and exclude the most important differential diagnoses of prolactinomas.
\end{abstract}

\section{Keywords}

Prolactin, Hyperprolactinemia, Macroprolactinemia, Prolactinomas, PEG

\section{Abbreviations \\ PRL: Prolactin; PRL-R: Prolactin Receptor; MacroPRL: Macroprolactin; HyperPRL: Hyperprolactinemia; CKD: Chronic Kidney Disease; Prolactinomas: Prolactin- secreting Adenoma; PEG: Polyethylene Glycol; FSH: Follicle Stimulating Hormone; LH: Luteinizing Hormone; $\mathrm{AMH}$ : Anti-Mullerian Hormone}

\section{Introduction}

Prolactin (PRL) is a hormone synthesized and secreted by the anterior pituitary lactotrophs. This process is influenced by multiple stimulatory and inhibitory factors, the chief of which is dopamine [1]. Normally, dopamine from the hypothalamic arcuate nucleus acts on specific lactotroph dopamine receptors (D2) and inhibits pituitary PRL secretion [2]. The D2 receptor gene is located on chromosome 11 . PRL is encoded by a single gene, located on chromosome 6 in humans [3]. Following its production pre-PRL (227 amino acids) is cleaved to form PRL (199 amino acids), a $23 \mathrm{kDa}$ protein [4]. Extra-pituitary PRL has also been reported. Pituitary and extra-pituitary PRL have identical structures and bind PRL-R but the mechanisms of their regulation differ and are being elucidated [5]. Prolactin has strong structural homology with growth hormone and placental lactogen [6]. PRL is involved in the control of lactation. Post-partum, PRL stimulates the differentiation and proliferation on mammary cells that has been primed for lactation by other hormones - estrogen, progesterone, insulin, corticosteroids and Growth Hormone (GH). The large increase in PRL during lactation is associated with a decreased dopamine output, mediated by a decrease in the phosphorylation of tyrosine hydroxylase, the rate-limiting enzyme in dopamine synthesis [7]. During lactation, the increase in PRL is accompanied by the proliferation and hypertrophy of lactotrophs in the pituitary gland [8]. Even after lactation with a return of dopamine inhibition, the hypertrophy of the lactotrophs persists for several months, allowing for higher PRL levels with subsequent lactation [2]. 
Besides the $23 \mathrm{kDa}$ PRL monomer ('little PRL') other $P R L$ isoforms are present in the circulation. These macro-molecular complexes of PRL and IgG autoantibodies are 'big PRL' (48-56 kDa) and 'big big PRL' (> $150 \mathrm{kDa})$, a polymer referred to as macroprolactin (macro-PRL) [9]. In normal subjects, total circulating prolactin comprises $65-85 \%$ little PRL, $10-20 \%$ big prolactin, and $<10 \%$ big big prolactin [10]. Macro-PRL has minimal biological activity and pathological function but is variably detected in the current PRL immunoassays. The large size of macroPRL results in lower renal clearance and this elevates the total PRL without any increase in lactotroph PRL release. This phenomenon, also called "analytical hyperprolactinemia", can lead to misinterpretation of PRL results. Proteolytic cleavage of the active $23 \mathrm{kDa}$ PRL protein produces $14 \mathrm{kDa}, 16 \mathrm{kDa}$ and $22 \mathrm{kDa}$ PRL variants [4]. There has been increasing interest in the role of the $16 \mathrm{kDa}$ fragment of prolactin in angiogenesis. It uses plasminogen activator inhibitor- 1 as a binding partner to activate the urokinase-type plasminogen activator receptor [11,12] which inhibits angiogenesis [13]. This $16 \mathrm{kDa}$ PRL variant may have roles in peripartum cardiomyopathy [14] and preeclampsia [15].

PRL acts via its membrane receptor, the Prolactin Receptor (PRL-R), the gene for which is located on chromosome 5 [16]. PRL-R (598 amino acids) is formed from the cleavage of pre-PRL-R ( 622 amino acids). PRL-R is a member of the class 1 hematopoietic cytokine receptor superfamily that includes GH [17]. The structure of the PRL-R has now been elucidated and the mechanism of its activation deciphered [2]. Human PRL-R binds 3 ligands (PRL, GH, and placental lactogen) rendering it difficult to ascertain the specific effects of PRL in vivo [18]. The PRL-R, initially thought to be restricted to lactating cells, has been now found to be widespread in the body including in adipose tissue, skin and hair follicles, pancreas, bone and adrenal glands [19]. In the pancreas, it regulates the ontogenesis of pancreatic stem cells to create a functional reserve of beta cells [20] that may contribute to beta-cell proliferation during pregnancy.

The pituitary gland is an anatomic meshwork of differentiated cells with great plasticity that enables formation of new differentiated cell types in response to new signals and inputs [21]. Control of pituitary function is complex and finely integrated through three tiered loops - the hypothalamus, intrapituitary signals, and peripheral hormone feedback [22]. This complex regulation results in production of sufficiently abundant and appropriately timed regulatory secretions in response to external cues [23].

\section{Measurement of Serum Prolactin}

Current automated immunoassays employed in clinical laboratories use a two-site immunometric or sandwich assay principle. Prolactin in the sample reacts with a capture antibody immobilised on a solid phase and a labelled detection antibody. Following capture of the analyte-antibody sandwich, unreact- ed reagents are washed away. The signal generated is directly proportional to the sample prolactin concentration. These modern immunometric assays are free from cross-reactivity of related molecules such as growth hormone and placental lactogen. Interference from heterophilic antibodies are also very rarely encountered as such assays have been optimised with blocking agents. However, interference from macro-PRL remains common. Most of the PRL assays are standardised to the World Health Organisation's third international prolactin standard $84 / 500$, which consists of the $23 \mathrm{kDa}$ little PRL derived from human pituitaries. However, despite general concordance between PRL methods, agreement of PRL results from different vendors is still poor and PRL reference intervals are platform specific. The expected serum PRL concentration in normal adult males and females is less than $14 \mathrm{ug} / \mathrm{L}$ and $24 \mathrm{ug} / \mathrm{L}$ respectively (PRL conversion units: I ug/L $=21.2 \mathrm{mU} / \mathrm{L}$ ). The reported reference ranges for total PRL on the Abbott Architect assay is 2.7-19.7 ug/L for males and 3.0-26.4 ug/L for females [24]. Serum PRL is unaffected by food and a fasting sample is not necessary [25].

\section{Causes of Hyperprolactinemia}

The causes of hyperprolactinemia (hyperPRL) are listed in Table 1. The prevalence of hyperPRL is about 10 per 100,000 in men and 30 per 100,000 in women, respectively and it is the second most common cause of infertility in women after Polycystic Ovarian Syndrome (PCOS) [25].

Table 1: Causes of Hyperprolactinemia.

1. Analytical (e.g. Macroprolactinemia)

2. Physiological
a. Pregnancy
b. Lactation
c. Stress

3. Pharmacological

a. Anti-psychotics (e.g. risperidone)

b. Anti-hypertensives (e.g. verapamil)

c. Anti-depressants (e.g. tricyclic antidepressants)

d. Anti-epileptic drugs

e. Metoclopramide

f. Opiates

g. Estrogens (e.g. oral contraceptives)

4. Pathological

a. PRL secreting adenomas

b. Hypothalamic/pituitary stalk disorders (e.g. granulomatous disease, irridation, trauma)

c. Renal dysfunction

d. Hepatic failure

e. Hypothyroidism

f. Hereditary disorders

5. Idiopathic 


\section{Analytical HyperPRL or MacroPRL}

MacroPRL is a significant analytical cause of hyperPRL with a prevalence of between $12.5-40 \%$ in hyperPRL patients [26-28]. Contemporary PRL immunoassays are not able to discriminate macroPRL from monomeric PRL. Immunoassays often utilize a "sandwich" assay where a capture and a labelled antibody bind to different segments of the PRL molecule to generate a signal that is directly proportional to the amount of serum PRL. MacroPRL can still bind extensively with these antibodies and generate a high intensity signal for PRL despite not being biologically active. MacroPRL increases with age, is stimulated by dopamine antagonists and responds to the physiological stimuli that would normally increase PRL [9]. MacroPRL does not result in symptoms of hyperPRL. In fact, a recent study reported no evidence of hyperPRL symptoms in any of the 51 patients with macroPRL after a 10-year follow-up [29]. Although macroPRL is not of direct clinical significance, it may lead to misdiagnosis and injudicious management if not well understood [30]. MacroPRL can co-exist with true hyperPRL. In a study of 61 women with macroPRL and menstrual disturbances $59 \%$ had concomitant elevation of monomeric PRL after PEG precipitation [31]. Seemingly innocuous, laboratorians and clinicians need to be cognizant of the importance of macroPRL and hyperPRL [32]. Misdiagnosis can be avoided if the laboratory pretreats the serum sample with Polyethylene Glycol (PEG) to precipitate any macroprolactin before measuring prolactin [16].

\section{Physiologic HyperPRL}

There are physiologic causes for a rise in PRL such as pregnancy, lactation, physical exertion or stress. During pregnancy PRL increases in response to increasing estradiol ranging from 35-600 ug/L [33]. PRL is not increased in non-lactating women and men after nipple stimulation or breast imaging and breast examination $[34,35]$.

\section{Pharmacologic HyperPRL}

A variety of drugs (neuroleptics, antipsychotics e.g. risperidone, antidepressants, opiates, estrogens and metoclopramide) can cause hyperPRL [36]. Serum PRL is typically between $25-100 \mathrm{ug} / \mathrm{L}$ with the exception of risperidone where PRL levels up to $200 \mathrm{ug} / \mathrm{L}$ may be encountered [37]. A detailed drug history and a repeat PRL test after abstaining from the offending drug will exclude them as the cause of hyperPRL.

\section{Pathologic HyperPRL}

In Chronic Kidney Disease (CKD) PRL is elevated due to decreased clearance and increased secretion [38]. Some hypothyroid patients may have enlarged pituitary gland due to thyrotroph and/or lactotroph hyperplasia [39]. With treatment of hypothyroidism PRL values normalize. Among the most disconcerting causes of pathological hyperPRL are PRL secreting adenomas (prolactinomas) and hypothalamic/pituitary stalk disorders (including granulomatous disease, irradiation and trauma).

Prolactinomas account for up to $50 \%$ of the cases of hyperPRL [40]. The prevalence of pituitary adenoma ranges from $1 / 865-1 / 2688$ in the general population [41] with $40-46 \%$ of these being prolactinomas $[25,42]$. Prolactinomas are more commonly encountered in women [43]. Prolactinomas may be classified by size. Microadenomas $(<10 \mathrm{~mm})$ account for over $90 \%$ of cases [41]. Macroadenomas are over $>10 \mathrm{~mm}$ in size but may be greater than $40 \mathrm{~mm}$ (giant macroadenomas). PRL levels generally correspond to the tumour mass. Pituitary macroadenomas are typically associated with PRL levels $>250 \mathrm{ug} / \mathrm{L}$ [44]. In giant macroadenomas PRL may reach up to $>1000 \mathrm{ug} / \mathrm{L}$ [45]. The Endocrine Society highlights that PRL levels $>500 \mathrm{ug} / \mathrm{L}$ are highly suspicious of macroadenomas [25]. In a study by Vilar $(n=250)$ [40] the PRL levels in macroadenomas ranged from 100-> $500 \mathrm{ug} / \mathrm{L}$, but in only $35 \%$ of them was PRL $>500 \mathrm{ug} / \mathrm{L}$. As such, it is difficult to judge the size of prolactinomas based on PRL values alone. Larger prolactinomas can cause gonadotropin insufficiency from its compressive effects on the pituitary gland.

Most prolactinomas are sporadic. Occasionally, they occur as part of the rare hereditary disorders e.g. multiple endocrine neoplasia type 1 [46], Carney complex, or familial isolated macroprolactinoma [47]. Almost all prolactinomas are benign, but rarely they can be malignant and metastasize [48]. Ectopic prolactin secretion can also occur with ovarian dermoids or bronchogenic carcinoma [2].

\section{Idiopathic HyperPRL}

In some patients with PRL between 20-100 ug/L no cause can be identified although they may have microadenomas not visible on imaging. These cases are classified as idiopathic [49]. Familial cases of idiopathic hyperPRL have also been reported [50] from inactivating mutations in the PRL receptor. However, clinical manifestations of hyperPRL in these subjects were not manifest suggesting that loss-of-function mutation in the $\mathrm{PRL}$ receptor may result in inactive $\mathrm{PRL}$ isoforms or PRL insensitivity.

\section{Evaluation of Hyperprolactinemia}

\section{Symptoms}

Symptoms of true hyperPRL include galactorrhoea, secondary amenorrhea, oligomenorrhea and anovulatory infertility. While galactorrhoea is the classic symptom of hyperPRL, it only occurs in less than half of such cases [51]. HyperPRL inhibits neurons that release kisspeptin- $1[2,52]$. These neurons stimulate neurons in the hypothalamus to release Go- 
nadotropin-Releasing Hormone (GnRH). Thus, hyperPRL prevents $\mathrm{GnRH}$ and FSH/LH release resulting in hypogonadotropic hypogonadism. HyperPRL can also decrease estrogen levels through effects on ovarian aromatase activity and by blocking the stimulatory effects of FSH, as well as inhibit progesterone production [53]. HyperPRL in amenorrheic women with oestrogen deficiency aggravates osteoporosis. As PRL-R is present in bone [19], it is unclear if the low $B M D$ is due to hyperPRL or the low gonadotropins associated with hyperPRL. Larger adenomas cause gonadotropin insufficiency from its compressive effects on the pituitary gland. In men, hyperPRL can lead to hypogonadism, decreased libido, erectile dysfunction, infertility, gynecomastia and galactorrhea. Men often present with macroadenomas associated with visual symptoms and headaches, not just due to a diagnostic delay but also because males develop more aggressive tumors [54]. Males are thus at greater risk of hypopituitarism at diagnosis, with the gonadal and somatotropic axes more frequently affected than thyrotropic and corticotropic axes [55].

\section{Investigations}

Dynamic testing for PRL is not useful in the investigation of hyperPRL and is no longer recommended by the Endocrine Society [25]. For suspected hyperPRL, the Endocrine Society recommends a single measurement of serum PRL obtained at any time of the day [25]. Pregnancy should be excluded in women of child-bearing age since prolactinomas can co-exist with pregnancy. Besides, the stimulating effect of placental estrogens on pituitary adenomas can lead to local compressive symptoms [53]. Renal or liver impairment, hypothyroidism, and concomitant use of drugs that can cause hyperPRL should be ruled out. In amenorrheic women, Follicle-Stimulating Hormone (FSH), Luteinising Hormone (LH) and/or Anti-Mullerian Hormone (AMH) should also be assessed to exclude ovarian failure/polycystic ovarian syndrome [25]. Magnetic resonance imaging (MRI) should be performed to visualize pituitary/sellar masses, followed by biopsy for histological diagnosis. In males with hyperPRL, testosterone levels should also be evaluated. In addition, patients should also be assessed for acromegaly, as mixed-cell adenomas secreting prolactin and Growth Hormone (GH) can occur [56]. Typically, acromegalic patients only have a moderate degree of hyperPRL, with some studies showing PRL levels of $131.3 \pm 76.6 \mathrm{ug} / \mathrm{L}[57]$.

\section{Macroprolactinemia}

MacroPRL should be assessed in all hyperPRL samples. Size Exclusion Chromatography (SEC) remains the gold standard for confirming macroPRL, but it is labor intensive and impractical for regular laboratory use. An alternative common laboratory method is to use PEG precipitation, which is fast, convenient, and inexpen- sive. PEG solution is added to the plasma sample, mixed together, and centrifuged. MacroPRL and other macro-molecular PRLs are precipitated (PEG-precipitable PRL) and monomeric PRL measured on the supernatant (free PRL). Total PRL is measured on an unmanipulated sample. The amount of macroPRL is calculated thus:

\section{(Total PRL - free PRL) / Total PRL \%}

To diagnose macroPRL a PEG-precipitation ratio of > $60 \%$ or a post-PEG PRL recovery (free PRL/Total PRL) < $40 \%$ is used.

Despite the expediency of PEG precipitation, there is large variability in the diagnostic criteria for the presence of macroPRL after PEG precipitation. Different studies $[6,27,31]$ use different percentage PRL recovery cut-offs to diagnose the presence of macroPRL. Others [58] consider any decrease in PRL post-PEG treatment to be diagnostic. However, these percentage thresholds are arbitrary definitions and patients with recoveries below diagnostic cutoffs may still have true hyperPRL or macroPRL. It has been suggested that PEG recoveries of $30-65 \%$ be classified as indeterminate, requiring further analysis with other methods (e.g. SEC) to diagnose macroPRL. There are many different PEG precipitation protocols [59] with multiple sources of variation from sample volumes required, type of PEG used, PEG reconstitution methods, time of mixing, sample incubation and centrifugation time/temperature. This creates different recovery rates between laboratories and assays, which may further complicate the interpretation of PEG precipitation results as different amounts of true PRL are co-precipitated with the macroPRL depending on the PEG protocol.

Although macroPRL is not bioactive, non-specific symptoms of hyperPRL may still be present due to coincident elevation of PRL. If macroPRL is not recognized, it can lead to misdiagnosis and mismanagement of hyperPRL. In a study of 337 patients with hyperPRL [60], 88 patients (26\%) were found to have macroPRL. Some patients with macroPRL $(n=11)$ had abnormal pituitary MRI findings. In another study of 61 women with macroPRL and menstrual disturbances [31], 59\% had concomitant elevation of monomeric PRL after PEG precipitation and $36 \%$ of such patients had a pituitary abnormality on MRI imaging.

MacroPRL is a common occurrence in patients with hyperPRL. Contemporary PRL immunoassays are not able to discriminate macroPRL from monomeric $P R L$. There is also variation in PRL results on different assay platforms, particularly at higher concentrations of PRL, even after attempts at standardization with international reference materials. Different PRL assays vary in their ability to detect macroPRL: ranging from between 2.3 to 7.8 -fold difference [61] between the highest and lowest estimates. In our hospital, we have performed an analysis for macroPRL between 
two assays (Abbott Architect and Roche Cobas e602) [62]. Sixteen percent $(100 / 616)$ of the PRL requests were hyperPRL. The Architect PRL identified 18 subjects as macroPRL (18\%) while the Cobas PRL identified 12 subjects (12\%) although there was a $100 \%$ concordance of macroPRL between the 2 assays. It is thus preferable to monitor hyperPRL subjects using the same assay platform each time.

Most laboratories report results post-PEG precipitation as the percentage total prolactin recovered. However, this approach lacks diagnostic specificity especially when there is an excess of macroprolactin and monomeric prolactin at the same time. Thus, some studies have advocated the reporting of absolute $P R L$ values with a modified $P R L$ reference interval post-PEG precipitation to identify specimens with true hyperPRL after diagnosis of macroPRL [9,63-65]. $P R L$ reference intervals post-PEG treatment need to be assay specific and should be derived with normal samples similarly treated with PEG. Ideally larger populations can be used so that confidence intervals for reference intervals can be established. There are also rare instances of spurious results post PEG treatment, for example, elevated GGT levels can cause increased precipitation of monomeric PRL and a false-positive macroPRL result [9], whereas macroPRL containing IgA may not precipitate and give a false-negative result [66]. Non-lgG-type macroPRL may be significant sometimes as some studies [67] have reported a prevalence of up to $32.3 \%$ of some patients with macroPRL. This may account, in part, for the variability in the sensitivity of different assays to macroPRL.

To better screen for macroPRL with PEG precipitation, several strategies can be employed. The first strategy is to assess for macroPRL only in asymptomatic patients with hyperPRL [25]. The second strategy is PEG precipitation in those patients with moderately elevated PRL without identifiable cause [44]. The third strategy is all hyperPRL sera are screened for macroPRL $[9,68]$. We favour the reflex screening for macroPRL with PEG precipitation in all hyperPRL samples to save time, costs and inconvenience for the patient. Another approach is to use a level of PRL where we can confidently rule out the presence of macroPRL. This was also discussed in the latest AACE guidelines on macroPRL [28]. While the guideline recommends testing asymptomatic patients with elevated PRL, they cite a study [69] where PRL levels were $350-400 \mathrm{ug} / \mathrm{L}$ in a patient with macroPRL. Considering the large variation in amounts of macroPRL between hyperPRL patients, the guidelines had no recommendation for a level of PRL at which macroPRL testing can be safely omitted.

\section{Other prolactin assay pitfalls}

The hook effect is a factor which can affect PRL anal- ysis. In patients with giant pituitary adenomas, significantly high levels of PRL molecules saturate the antibodies of the sandwich assay, with signalling antibodies binding directly to excess PRL without capture antibodies, leading to results that are lower than expected [70]. This is an uncommon phenomenon that occurs in giant prolactinomas where the actual PRL levels can be as high as $1000-100,000 \mathrm{ug} / \mathrm{L}$ but are reported as $20-200$ ug/L [45]. The hook effect is important because the PRL levels are used to decide upon surgical or medical management in these giant prolactinomas. The hook effect can be overcome by serial dilution of serum specimens; a serial dilution to $1: 100$ is recommended particularly in male patients with a moderate PRL elevation [25].

Biotin is another interferent that can affect $P R L$ readings. Some automated immunoassays that incorporate biotin to label the capture antigen/antibody or the signal antibody are vulnerable. High serum biotin prevents the formation of biotin-antigen-antibody complexes, resulting in a falsely low signal and reading. However, such an aberrant PRL result depend on the amount of biotin intake. Biotin is also excreted by the kidneys, and their levels can increase in patients with renal dysfunction. Biotin levels in over-the-counter supplements are low (30-300 ug) and will not cause any assay interference. In one study [71] where samples were spiked with biotin, PRL readings only started to show significant decreases when biotin concentrations approached $500 \mathrm{ug} / \mathrm{L}$, a level only seen with high-dose biotin therapy (100-300 $\mathrm{mg} / \mathrm{d}$ ) for multiple sclerosis. At serum biotin concentrations of $500 \mathrm{ug} / \mathrm{L}$, the PRL decreased to $30.8-33.1 \%$ of baseline values. However, the prevalence of patients on significantly high levels of biotin is low. In a Mayo clinic study [72], only 2 out of 1944 emergency department patients had serum biotin concentrations > $100 \mathrm{ug} / \mathrm{L}$ (both patients had end stage renal disease).

Heterophilic or human anti-mouse antibodies can occasionally affect $\mathrm{PRL}$ readings in a biotin-like fashion [73]. No assay is completely fool proof and interferences should be considered when the PRL result is discordant with the clinical picture [74]. Care should also be taken in patients with autoimmune disease receiving antibody treatment, as many of these exogenous antibodies can induce the development of heterophilic/ human anti-mouse antibodies in these patients [74]. If antibodies are suspected, reanalysing the sample with an alternative method, serial dilution, antibody precipitation or antibody-blocking tubes can help to reduce the interference and provide a more accurate result.

If all investigations prove negative, the hyperPRL can be deemed idiopathic [75]. Such cases may be autoimmune in nature, as evidenced by the presence of anti-pituitary antibodies in some patients [76].

\section{Conclusion}

In conclusion, physicians must know how to ap- 
proach hyperPRL and exclude the most important differential diagnoses of prolactinomas. They must be aware of macroPRL. Detection of macroPRL with PEG is straightforward but not fool proof. All hyperPRL sera should be screened for macroPRL. Patients with hyperPRL can also be affected by the hook effect, biotin, or other interferents.

\section{References}

1. Grattan DR, Kokay IC (2008) Prolactin: a pleiotropic neuroendocrine hormone. J Neuroendocrinol 20: 752-763.

2. Bernard V, Young J, Chanson P, Binart N (2015) New insights in prolactin: pathological implications. Nat Rev Endocrinol 11: 265-275.

3. Truong AT, Duez C, Belayew A, Renard A, Pictet R, et al. (1984) Isolation and characterization of the human prolactin gene. EMBO J 3: 429-437.

4. Goffin V, Binart N, Touraine P, Kelly PA (2002) Prolactin: the new biology of an old hormone. Annu Rev Physiol 64: 47-67.

5. Marano RJ, Ben-Jonathan N (2014) Minireview: extrapituitary prolactin: an update on the distribution, regulation, and functions. Mol Endocrinol 28: 622-633.

6. Horseman ND, Yu-Lee LY (1994) Transcriptional regulation by the helix bundle peptide hormones: growth hormone, prolactin, and hematopoietic cytokines. Endocr Rev 15: 627-649.

7. Andrews ZB, Kokay IC, Grattan DR (2001) Dissociation of prolactin secretion from tuberoinfundibular dopamine activity in late pregnant rats. Endocrinology 142: 2719-2724.

8. Le Tissier PR, Hodson DJ, Martin AO, Romano N, Mollard $P(2015)$ Plasticity of the prolactin (PRL) axis: mechanisms underlying regulation of output in female mice. Adv Exp Med Biol 846: 139-162.

9. Fahie-Wilson M, Smith TP (2013) Determination of prolactin: the macroprolactin problem. Best Pract Res Clin Endocrinol Metab 27: 725-742.

10. Fahie-Wilson MN, John R, Ellis AR (2005) Macroprolactin; high molecular mass forms of circulating prolactin. Ann Clin Biochem 42: 175-192.

11. Macotela Y, Aguilar MB, Guzman-Morales J, Rivera JC, Zermeno C, et al. (2006) Matrix metalloproteases from chondrocytes generate an antiangiogenic $16 \mathrm{kDa}$ prolactin. J Cell Sci 119: 1790-1800.

12. Bajou K, Herkenne S, Thijssen VL, D'Amico S, Nguyen NQ, et al. (2014) PAl-1 mediates the antiangiogenic and profibrinolytic effects of $16 \mathrm{~K}$ prolactin. Nat Med 20: 741-747.

13. Struman I, Bentzien F, Lee H, Mainfroid V, D'Angelo G, et al. (1999) Opposing actions of intact and $\mathrm{N}$-terminal fragments of the human prolactin/growth hormone family members on angiogenesis: an efficient mechanism for the regulation of angiogenesis. Proc Natl Acad Sci USA 96: 1246-1251.

14. Hilfiker-Kleiner D, Sliwa K (2014) Pathophysiology and epidemiology of peripartum cardiomyopathy. Nat Rev Cardiol 11: $364-370$

15. Leanos-Miranda A, Campos-Galicia I, Ramirez-Valenzuela KL, Chinolla-Arellano ZL, Isordia-Salas I (2013) Circulating angiogenic factors and urinary prolactin as predictors of adverse outcomes in women with preeclampsia. Hypertension 61: 1118-1125.

16. Gibney J, Smith TP, McKenna TJ (2005) The impact on clinical practice of routine screening for macroprolactin. $\mathrm{J}$ Clin Endocrinol Metab 90: 3927-3932.

17. Arden KC, Boutin JM, Djiane J, Kelly PA, Cavenee WK (1990) The receptors for prolactin and growth hormone are localized in the same region of human chromosome 5. Cytogenet Cell Genet 53: 161-165.

18. Brooks CL (2012) Molecular mechanisms of prolactin and its receptor. Endocr Rev 33: 504-525.

19. Bernard V, Young J, Binart N (2019) Prolactin - a pleiotropic factor in health and disease. Nat Rev Endocrinol 15: 356365.

20. Auffret J, Freemark M, Carre N, Mathieu Y, Tourrel-Cuzin C, et al. (2013) Defective prolactin signalling impairs pancreatic $\beta$-cell development during the perinatal period. Am J Physiol Endocrinol Metab 305: 1309-1318.

21. Rizzoti K, Akiyama H, Lovell-Badge R (2013) Mobilized adult pituitary stem cells contribute to endocrine regeneration in response to physiological demand. Cell Stem Cell 13: 419-432.

22. Le Tissier $P$, Campos $P$, Lafont $C$, Romanò $N$, Hodson DJ, et al. (2017) An updated view of hypothalamic-vascular-pituitary unit function and plasticity. Nat Rev Endocrinol 13: 257-267.

23. Budry L, Lafont C, El Yandouzi T, Chauvet N, Conéjero G, et al. (2011) Related pituitary cell lineages develop into interdigitated 3D cell networks. Proc Natl Acad Sci U S A 108: 12515-12520.

24. Whitehead SJ, Cornes MP, Ford C, Gama R (2015) Reference ranges for serum total and monomeric prolactin for the current generation Abbott Architect assay. Ann Clin Biochem 52: 61-66.

25. Melmed S, Casanueva FF, Hoffman AR, Kleinberg DL, Montori VM, et al. (2011) Diagnosis and treatment of hyperprolactinemia: an Endocrine Society clinical practice guideline. J Clin Endocrinol Metab 96: 273-288.

26. Alpanes M, Sanchon R, Martinez-Garcia MA, Martinez-Bermejo E, Escobar-Morreale HF (2013) Prevalence of hyperprolactinaemia in female premenopausal blood donors. Clin Endocrinol (Oxf) 79: 545-549.

27. Hattori N, Ishihara T, Saiki Y (2009) Macroprolactinaemia: prevalence and aetiologies in a large group of hospital workers. Clin Endocrinol (Oxf) 71: 702-708.

28. Samson SL, Hamrahian AH, Ezzat S, AACE Neuroendocrine and Pituitary Scientific Committee, American College of Endocrinology (ACE) (2015) American Association of Clinical Endocrinologists, and American College of Endocrinology disease state clinical review: clinical relevance of macroprolactin in the absence or presence of true hyperprolactinemia. Endocr Pract 21: 1427-1435.

29. Wallace IR, Satti N, Courtney $\mathrm{CH}$, Leslie H, Bell PM, et al. (2010) Ten-year clinical follow-up of a cohort of 51 patients with macroprolactinemia establishes it as a benign variant. J Clin Endocrinol Metab 95: 3268-3271.

30. Levy A (2014) Interpreting raised serum prolactin results. BMJ 348: 3207.

31. Lewandowski KC, Gasior-Perczak D, Kowalska A, Lewinski A (2014) Coexistence of macroprolactinaemia and hyperprolactinaemia in women with oligo-/amenorrhoea is associated with high risk of pituitary adenomas. Gynecol Endocrinol 30: 385-387.

32. Kasum M, Pavicic-Baldani D, Stanic $P$, Oreskovic $S$, Saric JM, et al. (2014) Importance of macroprolactinemia in hyperprolactinemia. Eur J Obstet Gynecol Reprod Biol 183: 28-32. 
33. Tyson JE, Hwang P, Guyda H, Friesen HG (1972) Studies of prolactin secretion in human pregnancy. Am J Obstet Gynecol 113: 14-20.

34. Sarac F, Tutuncuoglu P, Ozgen AG, Saygili F, Yilmaz C et al. (2008) Prolactin levels and examination with breast ultrasound or mammography. Adv Ther 25: 59-66.

35. Jarrell J, Franks S, Mclnnes R, Gemayel K, Guyda H, et al. (1980) Breast examination does not elevate serum prolactin. Fertil Steril 33: 49-51.

36. Molitch ME (2008) Drugs and prolactin. Pituitary 11: 209218.

37. Kearns AE, Goff DC, Hayden DL, Daniels GH (2000) Risperidone-associated hyperprolactinemia. Endocr Pract 6: 425-429.

38. Sievertsen GD, Lim VS, Nakawatase C, Frohman LA (1980) Metabolic clearance and secretion rates of human prolactin in normal subjects and in patients with chronic renal failure. $\mathrm{J}$ Clin Endocrinol Metab 50: 846-852.

39. Grubb MR, Chakeres D, Malarkey WB (1987) Patients with primary hypothyroidism presenting as prolactinomas. Am J Med 83: 765-769.

40. Vilar L, Freitas MC, Naves LA, Casulari LA, Azevedo M, et al. (2008) Diagnosis and management of hyperprolactinemia: results of a Brazilian multicenter study with 1234 patients. J Endocrinol Invest 31: 436-444.

41. Molitch ME (2017) Diagnosis and Treatment of Pituitary Adenomas: A Review. JAMA 317: 516-524.

42. Gruppetta M, Mercieca C, Vassallo J (2013) Prevalence and incidence of pituitary adenomas: a population based study in Malta. Pituitary 16: 545-553.

43. Iglesias P, Díez JJ (2013) Macroprolactinoma: a diagnostic and therapeutic update. QJM 106: 495-504.

44. Casanueva FF, Molitch ME, Schlechte JA, Abs R, Bonert V, et al. (2006) Guidelines of the Pituitary Society for the diagnosis and management of prolactinomas. Clin Endocrinol (Oxf) 65: 265-273.

45. Maiter D, Delgrange E (2014) Therapy of endocrine disease: the challenges in managing giant prolactinomas. Eur J Endocrinol 170: R213-227.

46. Verges B, Boureille F, Goudet $P$, Murat A, Beckers A, et al. (2002) Pituitary disease in MEN type 1 (MEN1): data from the France-Belgium MEN1 multicenter study. J Clin Endocrinol Metab 87: 457-465.

47. Raverot G, Arnous W, Calender A, Trouillas J, Sassolas $\mathrm{G}$, et al. (2007) Familial pituitary adenomas with a heterogeneous functional pattern: clinical and genetic features. $J$ Endocrinol Invest 30: 787-790.

48. Walker JD, Grossman A, Anderson JV, Ur E, Trainer PJ, et al. (1993) Malignant prolactinoma with extracranial metastases: a report of three cases. Clin Endocrinol (Oxf) 38 : 411-419.

49. Sluijmer AV, Lappöhn RE (1992) Clinical history and outcome of 59 patients with idiopathic hyperprolactinemia. Fertil Steril 58: 72-77.

50. Newey PJ, Gorvin CM, Thakker RV (2014) Mutant prolactin receptor and familial hyperprolactinemia. N Engl J Med 370: $977-978$.

51. Majumdar A, Mangal NS (2013) Hyperprolactinemia. J Hum Reprod Sci 6: 168-175.

52. Sonigo C, Bouilly J, Carre N, Tolle V, Caraty A, et al. (2012)
Hyperprolactinemia-induced ovarian acyclicity is reversed by kisspeptin administration. J Clin Invest 122: 3791-3795.

53. Molitch ME (2015) Endocrinology in pregnancy: management of the pregnant patient with a prolactinoma. Eur J Endocrinol 172: 205-213.

54. Schaller B (2005) Gender-related differences in prolactinomas. A clinicopathological study. Neuro Endocrinol Lett 26: 152-159.

55. Iglesias P, Bernal C, Villabona C, Castro JC, Arrieta F, et al. (2012) Prolactinomas in men: a multicentre and retrospective analysis of treatment outcome. Clin Endocrinol (Oxf) 77: $281-287$

56. Bonert VS, Melmed S (2006) Acromegaly with moderate hyperprolactinemia caused by an intrasellar macroadenoma. Nat Clin Pract Endocrinol Metab 2: 408-412.

57. Wang M, Mou C, Jiang M, Han L, Fan S, et al. (2012) The characteristics of acromegalic patients with hyperprolactinemia and the differences in patients with merely GH-secreting adenomas: clinical analysis of 279 cases. Eur J Endocrinol 166: 797-802.

58. Suliman AM, Smith TP, Gibney J, McKenna TJ (2003) Frequent misdiagnosis and mismanagement of hyperprolactinemic patients before the introduction of macroprolactin screening: application of a new strict laboratory definition of macroprolactinemia. Clin Chem 49: 1504-1509.

59. Favresse J, Bastin P, Fillee C, Luyckx F, Maiter D, et al. (2017) Tracking macroprolactin: use of an optimized polyethylene glycol precipitation method more compatible with the requirements and processes of automated core laboratories. J Appl Lab Med 1: 661-667.

60. Isik S, Berker D, Tutuncu YA, Ozuguz U, Gokay F, et al. (2012) Clinical and radiological findings in macroprolactinemia. Endocrine 41: 327-333.

61. Smith TP, Sulliman AM, Fahie-Wilson MN, McKenna TJ (2002) Gross variability in the detection of prolactin in sera containing big big prolactin (macroprolactin) by commercial immunoassays. J Clin Endocrinol Metab 87: 5410-5415.

62. Wee LYC, Liang YL, Yew YF, Aw TC (2018) Investigating Macroprolactin in a tertiary care hospital. $70^{\text {th }}$ AACC Annual Scientific Meeting Abstracts A-174: S59.

63. McCudden CR, Sharpless JL, Grenache DG (2010) Comparison of multiple methods for identification of hyperprolactinemia in the presence of macroprolactin. Clin Chim Acta 411: 155-160.

64. Smith TP, Fahie-Wilson MN (2010) Reporting of post-PEG prolactin concentrations: time to change. Clin Chem 56: 484-485.

65. Beltran L, Fahie-Wilson MN, McKenna TJ, Kavanagh L, Smith TP (2008) Serum total prolactin and monomeric prolactin reference intervals determined by precipitation with polyethylene glycol: evaluation and validation on common immunoassay platforms. Clin Chem 54: 1673-1681.

66. Ram S, Harris B, Fernando JJ, Gama R, Fahie-Wilson M (2008) False-positive polyethylene glycol precipitation tests for macroprolactin due to increased serum globulins. Ann Clin Biochem 45: 256-259.

67. Hattori N, Aisaka K, Shimatsu A (2016) A possible cause of the variable detectability of macroprolactin by different immunoassay systems. Clin Chem Lab Med 54: 603-608.

68. McKenna TJ (2009) Should macroprolactin be measured in all hyperprolactinaemic sera? Clin Endocrinol (Oxf) 71: 466-469. 
69. Jackson RD, Wortsman J, Malarkey WB (1985) Macroprolactinemia presenting like a pituitary tumor. Am J Med 78: 346-350.

70. Petakov MS, Damjanovic SS, Nikolic-Durovic MM, Dragojlovic ZL, Obradovic S, et al. (1998) Pituitary adenomas secreting large amounts of prolactin may give false low values in immunoradiometric assays: the hook effect. J Endocrinol Invest 21: 184-188.

71. Trambas C, Lu Z, Yen T, Sikaris K (2018) Characterization of the scope and magnitude of biotin interference in susceptible Roche Elecsys competitive and sandwich immunoassays. Ann Clin Biochem 55: 205-215.

72. Katzman BM, Lueke AJ, Donato LJ, Jaffe AS, Baumann NA (2018) Prevalence of biotin supplement usage in outpa- tients and plasma biotin concentrations in patients presenting to the emergency department. Clin Biochem 60: 11-16.

73. Sapin R, Simon C (2001) False hyperprolactinemia corrected by the use of heterophilic antibody-blocking agent. Clin Chem 47: 2184-2185.

74. Bolstad N, Warren DJ, Nustad K (2013) Heterophilic antibody interference in immunometric assays. Best Pract Res Clin Endocrinol Metab 27: 647-661.

75. Glezer A, Bronstein MD (2012) Approach to the patient with persistent hyperprolactinemia and negative sellar imaging. J Clin Endocrinol Metab 97: 2211-2216.

76. De Bellis A, Colao A, Pivonello R, Savoia A, Battaglia M, et al. (2007) Antipituitary antibodies in idiopathic hyperprolactinemic patients. Ann N Y Acad Sci 1107: 129-135. 